\title{
Hinweise für Autoren
}

Bitte senden Sie Ihr Manuskript direkt an den Verlag S. Karger.

Das Manuskript soil in druckreifem Zustand in dreifacher Ausfertigung eingereicht werden. Die Entscheidung über die Aufnahme erfolgt aufgrund mehrerer Gutachten. Eine Kopie des Manuskripts soil grundsätzlich beim Autor verbleiben.

Die typographische Gestaltung und redaktionelle Bearbeitung ist dem

Verlag vorbehalten.

Autorkorrekturen über 10\% der Satzkosten hat der Autor zu tragen.

Dem Autor stehen 40 Gratissonderdrucke seiner Arbeit zu.

Verlagsrecht. Mit der Annahme des Manuskriptes gehen alle Rechte an Sonder- und

Nachdrucken, an Abdrucken in anderen Publikatio-nen sowie an Übersetzungen auf den Verlag über. Ebenso erhält der Verlag das Recht von Vervielfältigungen (auch von Abbildungen) jeder Art wie auch Vortrag, Funk, Fernsehen sowie Speicherung in Datenverarbeitungsanlagen - auch von Teilen eines Manuskripts.

Markennamen müssen, wenn sie geschützt sind, mit einem hochge-stellten ${ }^{\circledR}$ bezeichnet werden. Die Verantwortung für die korrekte Befolgung dieser Vorschrift liegt ausschließlich beim Autor. Abkürzungen sollen möglichst sparsam verwendet werden. Bei spe-ziellen Abkürzungen sollte der Autor jeweils beim ersten Auftreten das Wort ausschreiben und die später verwendete Abkürzung in Klammern folgen lassen: z.B. Adenosin-Monophosphat (AMP).

Institutsangabe in der Sprache der Arbeit.

Zusammenfassungen sollen 20 Schreibmaschinenzeilen nicht über-schreiten. Eine Fassung in englischer Sprache und der englische Titel der Arbeit müssen mitgeliefert werden, auch wenn die Arbeit in deutscher Sprache abgefaßt ist. In die Zusammenfassung gehören keine Literaturzitate, Tabellen, Abbildungen und Abbildungshinweise sowie allgemein gehaltene Aussagen.

Schlüsselwòrter sollen ebenfalls in deutscher und englischer Sprache abgefaßt werden. Zu einer Arbeit gehören drei bis fünf Key Words.

Tabellen, Abbildungen und Literaturzitate müssen im Text erwähnt werden.

Abbildungsvorlagen dürfen nicht aufgeklebt, gelocht oder mit Büro-klammern angeheftet eingereicht werden und sollen auf der Rückseite den Namen des Autors, den Titel des Beitrags sowie die Bildnummer tragen.

Adresse des Autors ist am Schluß der Arbeit vollständig anzugeben.

Literaturverzeichnis. Es soil nur die im Text zitierten Publikationen, diese dafür lückenlos, enthalten. Die Literatur ist entweder alphabe-tisch zu ordnen oder entsprechend der Reihenfolge im Text zu nume-rieren.

\section{Sonderbände}

Die nachfolgend aufgeführten Sonderbände sind nur in beschränkter Auflage vorrätig. Der Preis ist abhängig vom Umfang. Abonnenten erhalten diese zu einem um $20 \%$ vom Ladenpreis ermäßigten Vorzugspreis. Bisher sind erschienen: 


\section{Beiträge zur Urologie}

(Reihenherausgeber E. Schmiedt und H.-W. Bauer, München)

Sonderband 1: Diagnostik und Therapie des Prostatakarzinoms.

Symposium der Urologischen Universitätsklinik München in

Zusammenarbeit mit dem Tumorzentrum München, München,

November '78

168 S., 67 Abb., 48 Tab., brosch., DM 40-

Sonderband 2: Berührungsfreie Nierensteinzertrümmerung

durch extrakorporal erzeugte, fokussierte Stoßwellen.

November 1980

98 S., 56 Abb., 8Tab., brosch., DM60-

Beiträge zur Onkologie

Sonderband 1: Chemotherapie urologischer Malignome. Prostata, Harnblase, Penis/Harnröhre.

Seminar des Berufs-verbandes Deutscher Urologen e.V, Mainz, März 1979190 S., 70 Abb., 59

Tab., brosch., DM 35-

Sonderband 2: Chirurgische Therapie des Mammakarzinoms. 1. Österreichische

Chirurgentagung, Salzburg, September 1978. VIII, 60 S., 16 Abb., brosch., DM 25,-

Sonderband 3: CISPLATIN. Derzeitiger Stand und neue Ent-wicklungen in der Chemotherapie maligner Neoplasien. Symposium am 16. November 1979, Frankfurt a. M. VIII, 176 S., 37 Abb., 89 Tab., brosch., DM 35-Sonderband 4: Probleme der Krebsnachsorge. Prognose, Begut-achtung und Rehabilitation bei gynäkologischen Karzinomen. VIII, 140S., 32 Abb., 13Tab., brosch.,

\section{DM40-}

Sonderband 5: Neue Erfahrungen mit Oxazaphosphorinen unter besonderer Berücksichtigung des Uroprotektors Uromitexan ${ }^{\circledR}$.

Gemeinsames Symposium der Arbeitsgemeinschaft für inter-

nistische Onkologie (AIO) der Deutschen Krebsgesellschaft und der ASTA-Werke AG, Bielefeld, am 29. Februar 1980 in

Düsseldorf.

X, 126 S., 36 Abb., 49 Tab., brosch., DM 35-

Sonderband 6: Proceedings of the International Vinca Alkaloid

Symposium - Vindesine.

Symposium November 1980, Frankfurt a. M.

XII, 460 S., 52 Abb., 208 Tab., brosch., DM 95 -

Sonderband 7: Critical Evaluation of Tumor Markers.

VIII, 120 S., 30 Abb., 24 Tab, brosch, DM 40-

Sonderband 8: Nicht-seminomatöse Hodentumoren.

AlO-Symposium Februar 1981, Bad Neuenahr.

X, 298 S., 113 Abb.,107 Tab., brosch., DM60,-

Sonderband 9: Adriamycin-Symposium -

Ergebnisse und Aspekte.

X, 462 S., 110 Abb. + 5 Farbtf., 167 Tab., brosch., DM 95-

Sonderband 10: Plasmapheresis in Immunology and Oncology.

Al/-Symposium December 1980, Göttingen

VIII, 288 S., 101 Abb., 44 Tab., brosch., DM 70 -

Sonderband 11: Interferon-Properties, Mode of Action,

Production, Clinical Application 
3rd International Expert Meeting March 1981, Bonn

X,234 S., 50 Abb., 70 Tab., brosch., DM 60-

Sonderband 12: Wirkung und Nebenwirkungen von Bleomycin -

Klinische und experimentelle Ergebnisse.

AlO-Symposium Oktober 1981, Hamburg

X, 220 S., 62 Abb., 91 Tab., 9 Farbtafeln, brosch., DM 65-

Sonderband 13: Aktuelle Probleme der Hämatologieund Onkologie.

Jahreskongreß der Dt. Ges. für Hämatologie und Onkologie,

Dezember 1981, München

XII, 387 S.,71 Abb., 106Tab., brosch., DM95,-

Sonderband 14: Tegafur/Ftorafur ${ }^{\circledR}$. Proceedings of the International Tegafur Workshop, Vienna 1982 VII, 145 S., 37 Abb., 34 Tab., brosch., DM 55- 